\title{
Clinical and surgical complexity in severe osteoporosis
}

\author{
Giovanni Iolascon $^{1} \cdot$ Umberto Tarantino $^{2}$
}

Received: 25 May 2015/Accepted: 4 June 2015/Published online: 8 September 2015

(C) Springer International Publishing Switzerland 2015

Demographic and epidemiological changes in recent decades have brought osteoporosis and related fragility fractures to be an emerging clinical condition [1]. Osteoporotic patients are old and affected by several comorbidities. As a consequence, they present a clinical complex context that would require a "person centered care" approach involving both medical and social interventions [2]. This is particularly true in patients with severe osteoporosis that is identified as the reduced bone density, with a T-score value below the $-2.5 \mathrm{SD}$ determined by dual energy X-ray absorptiometry, and the presence of one or more fragility fractures [3]. This definition does not really reflect the spectrum of severity of the disease that determines a variable increase in fracture risk [4]. The management of the patient with severe osteoporosis is complicated by his/ her multimorbidity (about $50 \%$ of the over 65 aged people takes more than 5 different drugs daily) [5] making both pharmacological and surgical approaches more challenging. For example, individuals with hip fracture are typically complex patients, with coexisting comorbidities, disability and frailty [6]. A study investigating the effect of comorbidities on mortality after hip fracture showed that in elderly people, the presence of 3 or more comorbidities was the strongest preoperative risk factor [7]. The aim of the treatment is to bring these patients back to their prefracture functioning level, and to their former activities and

Giovanni Iolascon

giovanni.iolascon@gmail.com

1 Department of Medical and Surgical Specialties and Dentistry, Second University of Naples, Naples, Italy

2 Department of Orthopaedics and Traumatology, University of Rome Tor Vergata, University Hospital Foundation Policlinico Tor Vergata, Rome, Italy social participation as soon as possible, and the orthopedic surgeon should perform a proper management of osteoporosis taking into account the patient's frailty. The concomitance of several diseases, among which it is sometimes difficult to identify the most relevant to prognosis, the therapeutic approach, and the consumption of health care resources are actually defined as "multimorbidity" [8].

The multidimensional interaction of different clinical conditions, in terms of comorbidities and intensity of care required in a single patient, is defined as "complexity" [2].

In science, a complex system is characterized by a large number of interacting factors. In medicine, the interactions among these factors could lead to high risk for health issues.

Clinical complexity is primarily based on the assessment and on the stratification of high clinical risk. It is related to the whole complex disease, including diagnostic approaches, healthcare strategies, organizational issues and therapeutic interventions, which are proportionally sized to the complexity and the resources consumption. Surgical complexity should be ascribed to 3 factors: the overall clinical condition of the patient, the quality/quantity of bone tissue on which the surgical procedure is carried out, and the factors related to surgical technique and materials used. Fragility fractures typically involve all the above factors: osteoporotic patient is often old, comorbid, and frail, with poor bone strength that requires materials and surgical techniques specific for bone tissue with alterations of mechanical properties.

Therefore, the orthopedic management of patients with severe osteoporosis requires a specific and comprehensive clinical and surgical approach.

Several proposals to manage severe osteoporosis have been made in recent years, including Orthogeriatric Units 
and Fracture Liaison Services (FLS), which seem to have significantly improved the clinical outcome of patients with fragility fractures $[9,10]$.

The GISOOS (Italian Orthopedic Group for the Study of Severe Osteoporosis) aims to promote technical and scientific research in the field of severe osteoporosis and fragility fractures to improve the correct management of complex patients and establish new shared guidelines for both surgical and pharmacological treatment.

The 3rd National Congress of the GISOOS focused on the concept of complexity as a factor influencing the medical and surgical management of patients who sustained fragility fractures.

This supplement of "Aging Clinical and Experimental Research" is entirely dedicated to some clinical and surgical experiences in the management of complex patients in the Orthopedic Surgery Units in Italy.

\section{Compliance with ethical standards}

Conflict of interest None.

Ethical approval This article does not contain any studies with human participants or animals performed by any of the authors.

\section{References}

1. Tarantino U, Capone A, Planta M et al (2010) The incidence of hip, forearm, humeral, ankle, and vertebral fragility fractures in
Italy: results from a 3-year multicenter study. Arthritis Res Ther 12:R226

2. Mezzich JE, Salloum IM (2008) Clinical complexity and personcentered integrative diagnosis. World Psychiatry 7:1-2

3. (2003) Prevention and management of osteoporosis. World Health Organ Tech Rep Ser 921:1-164 (back cover)

4. Iolascon G, Resmini G, Gimigliano F (2011) Severe osteoporosis and its identification. Aging Clin Exp Res 23(2 Suppl):6-7

5. Guthrie B, Makubate B, Hernandez-Santiago V et al (2015) The rising tide of polypharmacy and drug-drug interactions: population database analysis 1995-2010. BMC Med 7:74

6. Tom SE, Adachi JD, Anderson FA Jr et al (2013) Frailty and fracture, disability, and falls: a multiple country study from the global longitudinal study of osteoporosis in women. J Am Geriatr Soc 61:327-334

7. Roche JJ, Wenn RT, Sahota O et al (2005) Effect of comorbidities and postoperative complications on mortality after hip fracture in elderly people: prospective observational cohort study. BMJ 331(7529):1374

8. Schäfer I, von Leitner EC, Schön G et al (2010) Multimorbidity patterns in the elderly: a new approach of disease clustering identifies complex interrelations between chronic conditions. PLoS One 5:e15941

9. Liem IS, Kammerlander C, Suhm N et al (2013) Identifying a standard set of outcome parameters for the evaluation of orthogeriatric co-management for hip fractures. Injury 44:1403-1412

10. Akesson K, Marsh D, Mitchell PJ et al (2013) Capture the fracture: a best practice framework and global campaign to break the fragility fracture cycle. Osteoporos Int 24:2135-2152 